Original Article (short paper)

\title{
Exercise Training Improves Heart Rate Recovery after Exercise in Hypertension
}

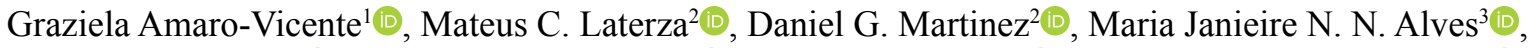 \\ Ivani C. Trombetta ${ }^{4}$, Ana Maria F. W. Braga ${ }^{3}{ }^{-}$, Edgar Toschi-Dias ${ }^{3}{ }^{\circledR}$, Maria Urbana P. B. Rondon ${ }^{1}$ \\ ${ }^{1}$ Universidade de Sao Paulo, USP, School of Physical Education and Sport, Sao Paulo, SP, Brazil, ${ }^{2}$ Universidade \\ Federal de Juiz de Fora, UFJF, Faculty of Physical Education and Sports, Juiz de Fora, MG, Brazil; \\ ${ }^{3}$ Instituto do Coração do Hospital das Clínicas da Faculdade de Medicina da Universidade de São Paulo \\ (InCor/HCFMUSP), São Paulo, Brazil.; ${ }^{4}$ Universidade Nove de Julho, UNINOVE, Sao Paulo, SP, Brazil
}

\begin{abstract}
Aim: This study tested the hypothesis that: 1- the exercise training would improve the heart rate recovery (HRR) decline after maximal exercise test in hypertensive patients and; 2- the exercise training would normalize HRR decline when compared to normotensive individuals. Methods: Sixteen hypertensive patients were consecutively allocated into two groups: Exercise-trained $(n=9,47 \pm 2$ years) and untrained ( $n=7,42 \pm 3$ years). An exercise-trained normotensive group $(n=11,41 \pm 2$ years $)$ was also studied. Heart rate was evaluated by electrocardiogram. The autonomic function was evaluated based on heart rate changes on the first and the second min of recovery after the maximal exercise test. Exercise training consisted of three 60-minute exercise sessions/week for 4 months. Results: In hypertensive patients, exercise training significantly increased the HRR decline in the first $(-19 \pm 2 v s .-34 \pm 3 \mathrm{bpm}, P=0.001)$ and second $(-33 \pm 3$ vs. $-49 \pm 2 \mathrm{bpm}, P=0.006)$ minutes after the maximal exercise test. In addition, after exercise training, the initial differences in the HRR decline after exercise between hypertensive patients and normotensive individuals were no longer observed (first minute: $-34 \pm 3$ vs. $-29 \pm 3 \mathrm{bpm}, P=0.52$, and second minute: $-49 \pm 2$ vs. $-47 \pm 4 \mathrm{bpm}, P=0.99$ ). Conclusion: Hypertension causes a delay in HRR after the maximal exercise test yet the exercise training normalizes HRR during the post-exercise period in hypertensive patients.
\end{abstract}

Keywords: autonomic modulation, exercise test, hypertensive patients, prognostic index.

\section{Introduction}

Heart rate recovery (HRR) defined as the difference in heart rate at peak exercise and at a specific time interval following the onset of recovery is an important index that has been used as a non-invasive method of assessment of cardiac autonomic recovery after exercise and has important clinical implications ${ }^{1-6}$. A diminished rate of heart rate decline during the first and second minutes of the recovery period after a maximal exercise test is associated with a poor prognosis in cardiovascular disease ${ }^{7-8}$. A prospective cohort study showed that low value for HRR after exercise testing is a powerful and independent predictor for all-cause mortality in patients with known or suspected of coronary heart disease ${ }^{9}$. Jouven, Empana, Schwartz, Desnos, Courbon, Ducimetiere ${ }^{10}$ reported that an HRR lower than 25 beats/min one minute after cessation of exercise conferred a relative risk of 2.2 for sudden death from myocardial infarction compared with the highest-percentile HRR group (higher than 40 beats $/ \mathrm{min})^{10}$.

One of the major pathophysiological mechanisms involved in hypertension is the autonomic dysfunction ${ }^{11-13}$. In fact, hypertension is associated with higher levels of sympathetic nerve activity ${ }^{14}$, decrease heart rate variability ${ }^{15}$ and delay $\mathrm{HRR}^{16-20}$. In hypertensive patients, it was shown a lower HRR after exercise and this autonomic dysfunction was related with blunting of the nighttime blood pressure (BP) fall ${ }^{16}$. Curiously, studies show that the reduction in the decline of HRR is observed even in the pre-hypertension stage ${ }^{17,21}$. Moreover, a recent study demonstrated that hypertensive patients under pharmacological treatment but with the levels of BP uncontrolled have a greater delay in $\mathrm{HRR}^{20}$.

On the other hand, exercise training has been recommended as a non-pharmacological therapy for the treatment of hypertension $^{22-24}$. And, the reduction in BP levels observed after exercise training has been associated with an improvement in the autonomic regulation in hypertensive patients ${ }^{25-26}$. It was recently demonstrated that patients after cardiac revascularization had an improvement in HRR decline after completion of a phase 2 cardiac rehabilitation program ${ }^{27}$. In addition, the authors observed that the patients with abnormal HRR who normalized HRR at the exit of rehabilitation program had improved survival compared with those who did not normalize the HRR ${ }^{27}$. However, the effects of exercise training on HRR in patients with hypertension are unknown. In addition, if the exercise training is able to normalize this index of cardiac autonomic function after exercise has not been documented in these patients.

Therefore, in the present investigation, we tested the hypothesis that: 1 - the exercise training would improve the 
HRR decline after maximal exercise test in hypertensive patients and; 2- the exercise training would normalize HRR decline when compared to normotensive individuals.

\section{Methods}

\section{Study Population}

Sixteen consecutive patients newly diagnosed and classified as stage 1 (systolic BP 140-159 mmHg and/or diastolic BP $90-99 \mathrm{mmHg})^{28}$ of hypertension were selected to participate in the study. An additional normotensive age-paired control group $(n=11)$ was also enrolled in the study. All subjects were sedentary, had no evidence of metabolic disorders, renal vascular hypertension, cerebral ischemic disease, or obstructive coronary artery disease at the time of the study. In addition, the subjects took no medication three months before the study. Those patients with a clinical indication of pharmacological treatment were excluded from our study to receive appropriate clinical treatment. The hypertensive patients were randomly divided into two groups: Exercise-trained patients $(\mathrm{n}=9)$ and untrained patients $(n=7)$. The normotensive control group was also submitted to exercise training. The calculated sample size was 7 patients in each group, taking into consideration previous publication ${ }^{17,27}$ with an alpha error of 0.05 and a power of 0.85 for HRR. At the end of the experimental protocol, the hypertensive patients of the control group were invited to participate in the exercisetraining program. All the participants gave their written informed consent. Some of the participants have been previously involved in other studies ${ }^{26,29}$. The University of São Paulo Medical School Ethical Committee approved the study for Human Research Protocols (no: 073/00).

\section{Measurements and Procedures}

\section{Clinical blood pressure}

The clinic BP levels were obtained with a mercury sphygmomanometer as previously described ${ }^{28}$. In summary, a minimum of three BP readings was taken on two separate days. Systolic and diastolic BP were recorded at the first appearance (phase I) and the disappearance (phase V) of Korotkoff sounds. The subjects were classified as hypertensive if the average of the systolic and diastolic BP levels were $\geq 140$ and/or $90 \mathrm{mmHg}$, respectively ${ }^{28}$.

\section{Exercise Testing}

Symptom-limited cardiopulmonary exercise testing was performed in an upright position on a bicycle ergometer under the supervision of a cardiologist. Subjects began cycling at an intensity of 0 Watt for a period of 2 minutes. Intensity was increased by 5,10 or 15 Watts every minute at 60 rotations per minute, followed by active recovery for a period of 2 minutes (Figure 1). During the recovery period, the first and second minutes were maintained at $50 \%$ and $25 \%$ of the maximal workload attained at the peak exercise test, respectively. All subjects were exercised to maximal exhaustion and exercise has never been stopped due to chest discomfort or electrocardiographic abnormalities. Heart rate was monitored continuously by 12-lead electrocardiogram (Cardio Control). BP was measured every 2 minutes with a column mercury sphygmomanometer.

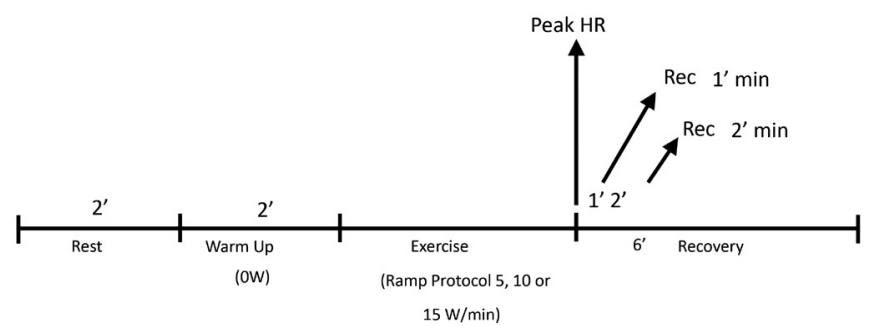

Figure 1. Timeline of experimental protocol (see Experimental Protocol section for more details).

\section{Functional Capacity Assessment}

Oxygen uptake and carbon dioxide production were determined by means of gas exchange on a breath-by-breath basis in a computerized system (CAD/Net 2001, Medical Graphics Corporation, St. Paul, MN, USA). Peak oxygen uptake was defined as the maximum attained oxygen uptake at the end of the exercise period in which the subject could no longer maintain the bicycle ergometer velocity at 60 rotations per minute. The anaerobic threshold was determined to occur at the point where there was a loss of linearity between oxygen uptake and carbon dioxide production or at the point where the ventilatory equivalent for oxygen or end-tidal oxygen partial pressure curves reached their respective minimum values and began to rise during the progressive exercise test ${ }^{30}$.

\section{Heart Rate Recovery}

HRR was evaluated by the difference between the heart rate achieved in the peak of exercise and the heart rate measured at the first minute of recovery, and by the difference between the heart rate achieved in the peak of exercise and heart rate measured at the second minute of recovery after the maximal exercise test performed on ergometric bicycle ${ }^{31}$.

\section{Exercise Training Program}

Subjects underwent exercise training under supervision at the Heart Institute. The 4-month training program consisted of three 60-minute exercise sessions per week. Each exercise session consisted of 5 minutes of stretching exercises, 40 minutes of 
cycling on a bicycle ergometer, 10 minutes of local strengthening exercises (sit-ups, push-ups, and pull-ups), and 5 minutes of cool down with stretching exercises. The exercise intensity was established by heart rate levels that corresponded with the anaerobic threshold up to $70 \%$ of peak oxygen uptake. The exercise session was started only if the systolic and diastolic BP levels at rest were lower than 160 and $105 \mathrm{mmHg}$, respectively ${ }^{32}$. Untrained patients were instructed to avoid any regular exercise program (supervised or unsupervised) for 4 months.

\section{Others Measurements}

Height $(\mathrm{m})$ and body weight $(\mathrm{kg})$ were measured using a calibrated scale. Based on these measures, the body mass index (BMI) was calculated, being expressed in $\mathrm{kg} / \mathrm{m}^{2}$.

\section{Experimental Protocol}

The study was performed at approximately 2:00 pm, with the subjects in a quiet air-conditioned room $\left(22^{\circ} \mathrm{C}\right.$ to $\left.24^{\circ} \mathrm{C}\right)$. The cardiopulmonary exercise test was repeated after 4 months in all subjects. In the exercise-trained group, this second evaluation was performed 48 hours after the last exercise session.

\section{Statistics}

Data are presented as mean \pm standard error. The unpaired $t$ test was used to test the baseline differences between hypertensive patients and normotensive individuals. A $\chi^{2}$ test was used to assess the gender differences between hypertensive patients and normotensive individuals. Two-way ANOVA (analysis of variance) with repeated measures was performed to test the differences among untrained hypertensive patients, exercisetrained hypertensive patients, and normal control individuals before and after the intervention. When a significant difference was found, Scheffé's post hoc comparison test was used. Significant differences were assumed to be at $P<0.05$.

\section{Results}

\section{Baseline Measurements}

Physical characteristics, hemodynamic measures, and maximal exercise testing data of the hypertensive patients and normotensive individuals are shown in Table 1. There were no significant differences in sex, age, height, weight, BMI, resting heart rate, peak heart rate, respiratory exchange ratio, and peak oxygen uptake between hypertensive patients and normotensive individuals. As expected, clinical systolic, diastolic and mean BP were significantly higher in hypertensive patients than in normotensive individuals.
Table 1. Baseline Physical Characteristics, Hemodynamic Measurements, and Maximal Exercise Testing Parameters in Hypertensive Patients and Normotensive Individuals

\begin{tabular}{lccc}
\hline & $\begin{array}{c}\text { Hypertensive } \\
\text { Patients } \\
(\boldsymbol{N}=\mathbf{1 6})\end{array}$ & $\begin{array}{c}\text { Normotensive } \\
\text { Individuals } \\
(\boldsymbol{N}=\mathbf{1 1})\end{array}$ & $\mathbf{P}$ \\
\hline Physical Characteristics & & & \\
Gender, Male/Female & $11 / 5$ & $9 / 2$ & 0.45 \\
Age, years & $42 \pm 3$ & $41 \pm 2$ & 0.25 \\
Height, m & $1.69 \pm 0.03$ & $1.67 \pm 0.02$ & 0.61 \\
Weight, kg & $73 \pm 5$ & $69 \pm 3$ & 0.33 \\
BMI, kg/m ${ }^{2}$ & $25.2 \pm 1.0$ & $24.7 \pm 0.9$ & 0.37 \\
\hline Hemodynamic Measurements & & & \\
Systolic BP, mmHg & $143 \pm 4$ & $119 \pm 2$ & $<0.001$ \\
Diastolic BP, mmHg & $93 \pm 2$ & $81 \pm 2$ & $<0.001$ \\
Mean BP, mmHg & $108 \pm 2$ & $94 \pm 2$ & $<0.001$ \\
HR, bpm & $78 \pm 4$ & $75 \pm 4$ & 0.52 \\
\hline Maximal Exercise Testing Parameters & & \\
Peak VO, mL/kg/min & $25.8 \pm 1.6$ & $29.1 \pm 2.1$ & 0.14 \\
Peak HR, bpm & $166 \pm 5$ & $175 \pm 4$ & 0.06 \\
Respiratory exchange ratio & $1.26 \pm 0.03$ & $1.25 \pm 0.03$ & 0.85 \\
\hline
\end{tabular}

Values are mean $\pm \mathrm{SE}$. $\mathrm{BMI}=$ body mass index; $\mathrm{BP}=$ blood pressure; $\mathrm{HR}=$ heart rate $\mathrm{VO}_{2}=$ oxygen uptake.

The decline rate of HRR in the first and second minutes after maximal exercise testing was significantly lower in hypertensive patients when compared with normotensive individuals (Fig. $2 \mathrm{~A}$ and $2 \mathrm{~B}$, respectively).

A

Absolute Change of Heart Rate Recovery in First Minute After Maximal Exercise Testing

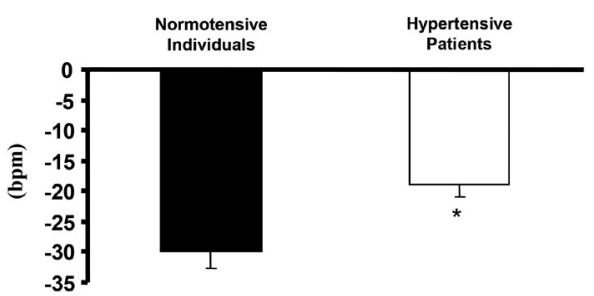

B

Absolute Change of Heart Rate Recovery in Second Minute After Maximal Exercise Testing

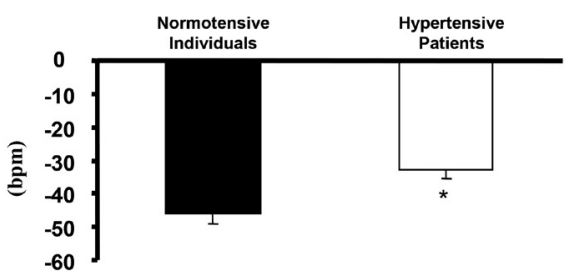

Figure 2. The decline rate of heart rate recovery (HRR) in first (Panel A) and second (Panel B) minutes after maximal exercise testing, in hypertensive patients and normotensive individuals. Note that the decline rate of HRR in first and second minutes after maximal exercise testing was significantly lower in hypertensive patients when compared with normotensive individuals. $*=$ significant difference between groups, $\mathrm{P}<0.05$. 


\section{Effects of Exercise Training Program}

Compliance with the exercise program was excellent, reaching an average of $96 \% \pm 3 \%$ and $96 \% \pm 3 \%$ of exercise sessions attended by both hypertensive patients and normotensive individuals, respectively. The effects of exercise training in exercise-trained hypertensive, untrained hypertensive, and exercise-trained normotensive groups are shown in Table 2. There were no significant difference among groups in weight,
BMI, peak heart rate, and peak respiratory exchange ratio pre and post-intervention.

Exercise training significantly decreased systolic, diastolic and mean BP in hypertensive patients. In addition, systolic, diastolic and mean BP levels were significantly lower in exercise-trained hypertensive patients than in untrained hypertensive patients, and similar to normotensive individuals (Table 2). Exercise training significantly decreased the baseline heart rate in hypertensive patients and normotensive individuals.

Table 2. Hemodynamic Measurements and Maximal Exercise Testing Parameters Pre and Post intervention in exercise-trained hypertensive, untrained hypertensive and exercise-trained normotensive groups.

\begin{tabular}{|c|c|c|c|c|c|c|}
\hline & \multicolumn{2}{|c|}{$\begin{array}{c}\text { Hypertensive } \\
\text { Exercise-Trained } \\
(\mathrm{N}=9)\end{array}$} & \multicolumn{2}{|c|}{$\begin{array}{c}\text { Hypertensive } \\
\text { Untrained } \\
(\mathrm{N}=7) \\
\end{array}$} & \multicolumn{2}{|c|}{$\begin{array}{c}\text { Normotensive } \\
\text { Exercise-Trained } \\
(\mathrm{N}=11)\end{array}$} \\
\hline & Pre & Post & Pre & Post & Pre & Post \\
\hline \multicolumn{7}{|l|}{ Physical Characteristics } \\
\hline Weight, kg & $74 \pm 4$ & $74 \pm 4$ & $73 \pm 6$ & $72 \pm 5$ & $69 \pm 3$ & $69 \pm 3$ \\
\hline BMI, $\mathrm{kg} / \mathrm{m}^{2}$ & $26.0 \pm 0.8$ & $26.2 \pm 0.9$ & $25.2 \pm 1.1$ & $24.7 \pm 1.0$ & $24.7 \pm 0.9$ & $24.6 \pm 0.9$ \\
\hline \multicolumn{7}{|l|}{ Hemodynamics Measurements } \\
\hline Systolic BP, mmHg & $143 \pm 3 *$ & $124 \pm 5^{\dagger}$ & $144 \pm 4 *$ & $143 \pm 6^{* \star}$ & $119 \pm 3$ & $117 \pm 3$ \\
\hline Diastolic BP, mmHg & $93 \pm 2 *$ & $81 \pm 3^{\dagger}$ & $94 \pm 2 *$ & $93 \pm 5^{* *}$ & $81 \pm 2$ & $77 \pm 2$ \\
\hline Mean BP, mmHg & $109 \pm 2 *$ & $94 \pm 2^{\dagger}$ & $109 \pm 2 *$ & $111 \pm 5^{\ddagger}$ & $94 \pm 2$ & $90 \pm 2$ \\
\hline Baseline HR & $81 \pm 5$ & $68 \pm 4^{\dagger}$ & $75 \pm 2$ & $74 \pm 4$ & $75 \pm 4$ & $66 \pm 3^{\dagger}$ \\
\hline \multicolumn{7}{|c|}{ Maximal Exercise Testing Parameters } \\
\hline Peak HR & $166 \pm 4$ & $168 \pm 4$ & $159 \pm 5$ & $156 \pm 6$ & $175 \pm 4$ & $173 \pm 5$ \\
\hline Peak respiratory exchange ratio & $1.25 \pm 0.02$ & $1.21 \pm 0.04$ & $1.26 \pm 0.04$ & $1.25 \pm 0.03$ & $1.25 \pm 0.03$ & $1.21 \pm 0.02$ \\
\hline
\end{tabular}

Values are mean $\pm \mathrm{SE}$. $\mathrm{BMI}=$ body mass index; $\mathrm{BP}=$ blood pressure; $\mathrm{HR}=$ heart rate. $*=$ Significant difference vs. normotensive individuals, $\mathrm{P}<0.05 ;{ }^{\dagger}=$ Significant difference pre- vs. post interventions, $\mathrm{P}<0.05 ;{ }^{*}=$ Significant difference vs. exercise-trained hypertensive patients, $\mathrm{P}<0.05$.

Exercise training significantly increased the decline rate of HRR in the first and second minutes after maximal exercise testing in exercise-trained hypertensive patients. In addition, at the end of the 4-month intervention period, the decline rate of HRR in the first and second minutes after maximal exercise testing was significantly higher in exercise-trained

A

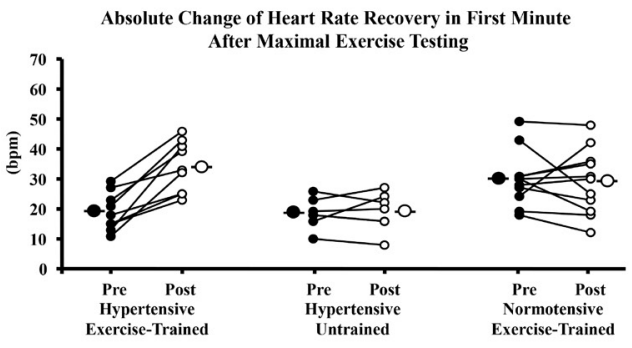

B

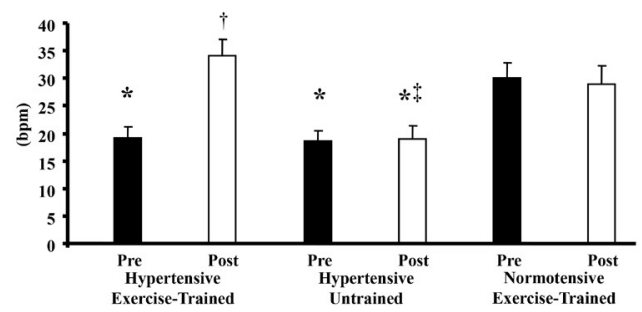

hypertensive patients than in untrained hypertensive patients $(P=0.001$ and $P=0.01$, respectively) and similar to normotensive individuals $(P=0.52$ and $P=0.99$, respectively, Fig. 3A and 3B). No significant changes in the decline rate of HRR in the first and second minutes were found in untrained hypertensive patients.
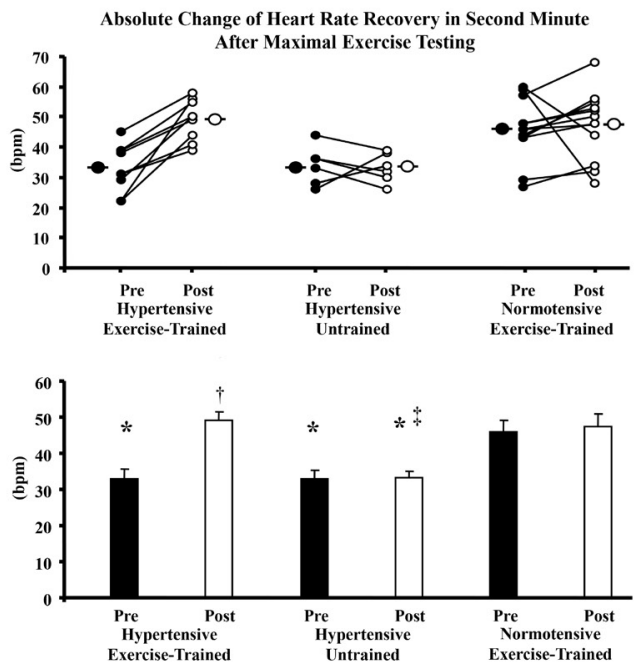

Figure 3. The decline rate of heart rate recovery (HRR) in first (Panel A and B) and second (Panel C and D) minutes after maximal exercise testing, in exercise-trained hypertensive patients, untrained hypertensive patients, and exercise-trained normotensive individuals. Note that exercise training significantly increased the decline rate of heart rate recovery (HRR) in first (Panel A and B) and second (Panel C and D) minutes after maximal exercise testing in hypertensive patients. $*=$ Significant difference vs. normotensive individuals; $\uparrow=$ Significant difference pré- vs. post interventions; $+=$ Significant difference vs. exercise-trained hypertensive patients, $\mathrm{P}<0.05$. 
Exercise training significantly increase peak oxygen uptake in hypertensive patients $(24.8 \pm 1.4 \mathrm{vs} .29 .5 \pm 2.3 \mathrm{~mL} / \mathrm{kg} / \mathrm{min}, P=0.01)$ and normotensive individuals $(29.1 \pm 2.1$ vs. $34.1 \pm 2.2 \mathrm{~mL} / \mathrm{kg} / \mathrm{min}$, $P=0.01)$. No significant changes were observed in peak oxygen uptake in untrained hypertensive patients over the 4-month duration of the study $(27.1 \pm 1.7$ vs. $26.5 \pm 1.3 \mathrm{~mL} / \mathrm{kg} / \mathrm{min}$, $P=0.79)$.

\section{Discussion}

The present study demonstrates that the decline rate of HRR in the first and second minutes after a maximal exercise test is reduced in hypertensive patients. In addition, our study shows that four months of exercise training restores the post-exercise decline rate of HRR in patients with high BP. These findings have important clinical implications. Firstly, diminished rate of heart rate decline after exercise suggests the presence of cardiac autonomic dysfunction and ${ }^{6-7,9,33}$ is indicative of poor prognosis ${ }^{8}$. Several studies have highlighted the prognostic role of the HRR index in humans ${ }^{7,9,10}$. In fact, previous observations suggest that healthy subjects with a an HRR lower than 25 beats per minute have 2.1 times the risk of sudden death and 1.3 times the risk of death from any cause than subjects with an HRR higher than 40 beats per minute ${ }^{10}$. In our study, we found that the decrease in heart rate at first minute of recovery of the termination of the exercise was $22 \pm 3$ beats per minute in hypertensive patients, which shows that these patients are under a higher risk of mortality. Secondly, exercise training significantly improves HRR in hypertensive patients. Moreover, this non-pharmacological strategy put the hypertensive patients in a lower risk zone of sudden death since the reduction of heart rate at first minute of recovery was $34 \pm 3$ beats per minute after the intervention. This improvement of HRR decline after exercise has been associated with a reduction of all-cause mortality. In fact, a recent study following 1070 patients after cardiac revascularization or cardiac surgery, who underwent exercise testing before and after 12 weeks cardiac rehabilitation, showed that patients with abnormal HRR at baseline, who had normalized HRR after rehabilitation program, had similar mortality to those with baseline normal HRR ${ }^{27}$. Additionally, the improvement of the HRR after exercise training program may have greater clinic relevance in patients who do not have an optimized control of BP levels even after pharmacological treatment. According to the $\mathrm{Li}$ et al. ${ }^{20}$ study, patients under pharmacological treatment but not controlled BP present an even greater delay in HRR compared with antihypertensive-controlled hypertensives ${ }^{20}$. However, new studies should be addressed to test this hypothesis.

The mechanisms by which exercise training increases HRR towards normal levels in hypertensive individuals are out of the scope of our study. However, previous information suggests that the decrease in heart rate after exercise can be attributed to amelioration in the cardiac vagal control. Some investigators reported that the decrease in heart rate after exercise was completely abolished by atropine administration ${ }^{2}$. Other information suggests that nitric oxide is involved in the cardiac vagal modulation after exercise. Sears, Choate,
Paterson $^{34}$ demonstrated that the nitric oxide synthase blockade with L-NMMA slowed the rate of decay in heart rate during vagal stimulation following adrenergic stimulation in the guinea pig $^{34}$. In addition, exercise training has been shown to increase nitric oxide levels in hypertensive patients ${ }^{35}$. Thus, we can speculate that exercise training increases cardiac vagal control in hypertensive patients, which may be due to higher nitric oxide bioavailability. We cannot rule out that the improvement in HRR is due to a reduction in sympathetic cardiac stimulation. In a recent study ${ }^{26}$, we reported that exercise training reduced muscle sympathetic nerve activity, which seemed to be associated with an enhancement in arterial baroreflex sensitivity. Despite the fact that we did not investigate sympathetic cardiac control in that study, it is reasonable to think that exercise training reduces sympathetic nerve activity not only in the blood vessel but also in the heart.

We and others have consistently demonstrated that moderate intensity exercise training, significantly reduces BP in patients with hypertension ${ }^{22-24,26,36-37}$. The present study confirms these observations since exercise training decreased systolic, diastolic and mean BP levels in hypertensive patients. Although the present study was not designed to evaluate the effects of exercise training on BP, we can speculate that an attenuation of sympathetic nervous activity may explain, at least in part, the reduction in BP levels observed in our trained hypertensive patients $^{26}$. Alternatively, hemodynamics ${ }^{38-41}$ and/or humoral ${ }^{42-43}$ mechanisms may also contribute to the reduction in BP after exercise training.

Resting bradycardia and and increase in peak oxygen consumption are good markers of exercise training adaptation ${ }^{44-45}$. Thus, the reduction in resting heart rate and the improvement in peak oxygen uptake after exercise training in hypertensive patients and in normotensive individuals demonstrates the effectiveness of exercise-training program.

The mechanisms involved in the reduction of resting heart rate after the exercise training, observed in the present study were not the scope of the present study. However it could be explained by a reduction in the cardiac sympathetic modulation, an increase in parasympathetic modulation to the heart or changes in the intrinsic heart rate r6-47 $^{4}$. Although we have not tested these mechanisms, previous studies in spontaneously hypertensive rats report that the resting bradycardia observed after exercise training in these animals is mediated by the reduction of sympathetic drive to the heart ${ }^{47}$.

On the other hand, the mechanisms that may explain the increase in peak oxygen consumption may be related to changes in cardiac output and arteriovenous oxygen difference. It is well documented that exercise training increases the arteriovenous oxygen difference by increasing capillary density, cardiac output, blood volume and peripheral oxygen extraction during exercise $^{48}$.

We recognize limitations in our study. We investigated patients in stage I of hypertension according to the national and international guidelines for the management of high BP in adults $^{23,28}$. Thus, our study provides no information regarding HRR in patients in stages II and III of hypertension in whom the impairment in HRR may be even more dramatic. Moreover, 
we do not know whether exercise training improves HRR in patients with more severe hypertension. This is an interesting topic for future investigations. Our exercise paradigm lasted four months. Thus, we do not know the training period needed for restoration of HRR in patients with hypertension. It is unlikely that the difference in HRR after exercise between exercise-trained hypertensive patients and untrained hypertensive patients was due to the peak heart rate achieved during the maximal exercise test, since there were no significant differences in peak heart rate between groups. However, the absence of medications reinforces our findings of the real effect of exercise training on HRR during the post-exercise period in this population. Moreover, is a useful tool to assess cardiac autonomic function in patients with hypertension, and the possible risk of sudden death in this population.

\section{Conclusion}

In conclusion, the exercise training improves the HRR decline after a maximal exercise test in hypertensive patients. In addition, HRR was normalized in these patients when compared with normotensive individuals. Therefore, our findings suggest a therapeutic effect of exercise training in the cardiac autonomic function in these patients.

\section{References}

1. Arai Y, Saul JP, Albrecht P, Hartley LH, Lilly LS, Cohen RJ, et al. Modulation of cardiac autonomic activity during and immediately after exercise. Am J Physiol. 1989; 256: H132-141.

2. Imai K, Sato H, Hori M, Kusuoka H, Ozaki H, Yokoyama H, et al. Vagally mediated heart rate recovery after exercise is accelerated in athletes but blunted in patients with chronic heart failure. J Am Coll Cardiol. 1994; 24: 1529-1535.

3. Goldberger JJ, Le FK, Lahiri M, Kannankeril JN, Kadish AH. Assessment of parasympathetic reactivation after exercise. Am J Physiol. 2006; 290: H2446-452.

4. Lahiri MK, Kannankeril PJ, Goldberger JJ. Assessment of autonomic function in cardiovascular disease: physiological basis and prognostic implications. J Am Coll Cardiol. 2008; 51: 1725-1733.

5. Peçanha T, Silva-Júnior ND, Forjaz CL. Heart rate recovery: autonomic determinants, methods of assessment and association with mortality and cardiovascular diseases. Clin Physiol Funct Imaging. 2014; 34: 327-339.

6. Peçanha T, Bartels R, Brito LC, Paula-Ribeiro M, Oliveira RS, Goldberger JJ. Methods of assessment of the post-exercise cardiac autonomic recovery: A methodological review. Int J Cardiol. 2017; 227: 795-802.

7. Nishime EO, Cole CR, Blackstone EH, Pashkow FJ, Lauer MS. Heart rate recovery and treadmill exercise score as predictors of mortality in patients referred for exercise ECG. JAMA. 2000; 284: 1392-1398.

8. Shetler K, Marcus R, Froelicher VF, Vora S, Kalisetti D, Prakash M, et al. Heart rate recovery: validation and methodologic issues. J Am Coll Cardiol. 2001; 38: 1980-1987.
9. Cole CR, Blackstone EH, Pashkow FJ, Snader CE, Lauer MS. Heart-rate recovery immediately after exercise as a predictor of mortality. N Engl J Med. 1999; 341: 1351-1357.

10. Jouven X, Empana JP, Schwartz PJ, Desnos M, Courbon D, Ducimetiere P. Heart-rate profile during exercise as a predictor of sudden death. N Engl J Med. 2005; 352: 1951-1958.

11. Chapleau MW, Li Z, Meyrelles SS, Ma X, Abboud FM. Mechanisms determining sensitivity of baroreceptor afferents in health and disease. Ann N Y Acad Sci. 2001; 940: 1-19.

12. Smith PA, Grahan LN, Mackintosh AF, Stoker JB, Mary DASG. Relationship between central sympathetic activity and stages of human hypertension. Am J Hypertens. 2004; 17: 217-222.

13. Schlaich MP, Lambert E, Kaye DM, Krozowski Z, Campbell DJ, Lambert G, et al. Sympathetic augmentation in hypertension; Role of nerve firing, norepinephrine reuptake, and angiotensina neuromodulation. Hypertension. 2004; 43: 169-175.

14. Grassi G, Ram VS. Evidence for a critical role of the sympathetic nervous system in hypertension. J Am Soc Hypertens. 2016; 10: 457-466.

15. Pagani M, Lucini D. Autonomic dysregulation in essential hypertension: insight from heart rate and arterial pressure variability. Auton Neurosci. 2001; 90: 76-82.

16. Polonia J, Amaral C, Bertoquini S, Martins L. Attenuation of heart rate recovery after exercise in hypertensive patients with blunting of the nighttime blood pressure fall. Int J Cardiol. 2006; 106: 238-243.

17. Erdogan D, Gonul E, Icli A, Yucel H, Arslan A, Akcay S, et al. Effects of normal blood pressure, prehypertension, and hypertension on autonomic nervous system function. Int J Cardiol. 2011; 151: 50-53.

18. Best SA, Bivens TB, Dean PM, Boyd KN, Melyn GM, Okada Y, et al. Heart rate recovery after maximal exercise is blunted in hypertensive seniors. J Appl Physiol. 2014; 117: 1302-1307.

19. Peçanha T, de Brito LC, Fecchio RY, de Sousa PN, da Silva Junior ND, de Abreu AP, et al. Metaboreflex activation delays heart rate recovery after aerobic exercise in never-treated hypertensive men. J Physiol. 2016; 594: 6211-6223.

20. Yu Y, Liu T, Wu J, Zhu P, Zhang M, Zheng W, et al. Heart rate recovery in hypertensive patients: relationship with blood pressure control. J Hum Hypertens. 2016; 31: 354-360.

21. Aneni E, Roberson LL, Shaharyar S, Blaha MJ, Agatston AA, Blumenthal RS, et al. Delayed heart rate recovery is strongly associated with early and late-stage prehypertension during exercise stress testing. Am J Hypertens. 2014; 27: 514-521.

22. Pescatello LS, Franklin BA, Fagard R, Farquhar WB, Kelley GA, Ray CA. American College of Sports Medicine position stand. Exercise and hypertension. Med Sci Sports Exerc. 2004; 36: 533-553.

23. James PA, Oparil S, Carter BL, Cushman WC, Dennison-Himmelfarb C, Handler J, et al. 2014 Evidence-Based guideline for the management of high blood pressure in adults: Report from the panel members appointed to the eighth joint national committee (JNC 8). JAMA. 2014; 31: 1809.

24. Whelton PK, Cary RM, Aronow WS, Casey Jr DE, Collins KJ, Himmelfarb DC, et al. 2017 ACC /AHA /AAPA /ABC /ACPM /AGS/ APhA/ ASH/ ASPC/ NMA/ PCNA Guideline for the Prevention, Detection, Evaluation, and Management of High Blood Pressure in Adults: A Report of the American College of Cardiology/American Heart Association Task Force on Clinical Practice Guidelines. JACC. 2017; 71: 1269-1324. 
25. Somers VK, Conway J, Johnston J, Sleight P. Effects of endurance training on baroreflex sensitivity and blood pressure in borderline hypertension. Lancet. 1991; 337: 1363-1368.

26. Laterza MC, de Matos LD, Trombetta IC, Braga AM, Roveda F, Alves MJ, et al. Exercise training restores baroreflex sensitivity in never-treated hypertensive patients. Hypertension. 2007; 49: 1298-1306.

27. Jolly MA, Brennan DM, Cho L. Impact of exercise on heart rate recovery. Circulation. 2011; 124: 1520-1526.

28. VII Diretriz Brasileira de Hipertensão. Arq Bras Cardiol. 2016; 107: 1-82.

29. Rondon MU, Laterza MC, de Matos LD, Trombetta IC, Braga AM, Roveda F, et al. Abnormal muscle metaboreflex control of sympathetic activity in never-treated hypertensive subjects. Am J Hypertens. 2006; 19: 951-957.

30. Skinner JS, McLellan TH. The transition from aerobic to anaerobic metabolism. Res Q Exerc Sport. 1980; 51: 234-248.

31. Cepeda FX, Toschi-Dias E, Maki-Nunes C, Rondon MU, Alves MJ, Braga AM, et al. Obstructive sleep apnea impairs postexercise sympathovagal balance in patients with metabolic syndrome. Sleep. 2015; 38: 1059-1066.

32. VI Diretrizes Brasileiras de Hipertensão. Arq Bras Cardiol. 2010; 95: 1-51.

33. Smith LL, Kukielka M, Billman GE. Heart rate recovery after exercise: a predictor of ventricular fibrillation susceptibility after myocardial infarction. Am J Physiol Heart Circ Physiol. 2005; 288:H1763-769.

34. Sears CE, Choate JK, Paterson DJ. Inhibition of nitric oxide synthase slows heart rate recovery from cholinergic activation. J Appl Physio.1 1998; 84: 1596-1603.

35. Higashi Y, Sasaki S, Kurisu S, Yoshimizu A, Sasaki N, Matsuura H, et al. Regular aerobic exercise augments endothelium-dependent vascular relaxation in normotensive as well as hypertensive subjects: role of endothelium-derived nitric oxide. Circulation. 1999; 100: 1194-1202.

36. Whelton SP, Chin A, Xin X, He J. Effect of aerobic on blood pressure: A meta-analysis of randomized, contrlled trials. Ann Intern Med. 2002; 136: 493-503.

37. Cornelissen VA, Smart NA. Exercise training for blood pressure: a systematic review and meta-analysis. J Am Heart Assoc. 2013; 2: e004473.

38. Amaral SL, Zorn TM, Michelini LC. Exercise training normalizes wall-to-lumen ratio of the gracilis muscle arterioles and reduces pressure in spontaneously hypertensive rats. J Hypertens. 2000; 18: $1563-1572$.

39. Amaral SL, Silveira NP, Zorn TM, Michelini LC. Exercise training causes skeletal muscle venular growth and alters hemodynamic responses in spontaneously hypertensive rats. J Hypertens. 2001; 19(5): 931-940.

40. Dinenno FA, Tanaka H, Monahan KD, Clevenger CM, Eskurza I, DeSouza CA, et al. Regular endurance exercise induces expansive arterial remodelling in the trained limbs of healthy men. J Physiol. 2001; 534(Pt 1): 287-295.

41. Almeida L, Freitas I, Souza L, Mira PAC, Martinez D, Laterza M. Condutância Vascular Aumentada em Indivíduos Fisicamente Ativos Filhos de Hipertensos. Rev Bras Ativ Fís Saúde. 2016; 21: 542-550.
42. Kiyonaga A, Arakawa K, Tanaka H, Shindo M. Blood pressure and hormonal responses to aerobic exercise. Hypertension. 1985; 7: $125-131$.

43. Felix JVC \& Michelini LC. Training-induced pressure fall in spontaneously hypertensive rats is associated with reduced angiotensionogen mRNA expression within the nucleus tractus solitari. Hypertension. 2007; 50: 780-785.

44. Hickson RC, Bomze HA, Holloszy JO. Linear increase in aerobic power induced by a strenuous program of endurance exercise. J Appl Physiol. 1977; 42: 372-376.

45. Katona PG, McLean M, Dighton DH, Guz A. Sympathetic and parasympathetic cardiac control in athletes and nonathletes at rest. J Appl Physiol. 1982; 52: 1652-1657.

46. Negrão CE, Moreira ED, Santos MC, Farah VM, Krieger EM. Vagal function impairment after exercise training. J Appl Physiol. 1992; 72: 1749-1753.

47. Gava NS, Véras-Silva AS, Negrão CE, Krieger EM. Low-intensity exercise training attenuates cardiac beta-adrenergic tone during exercise in spontaneously hypertensive rats. Hypertension. 1995; 26: 1129-1133

48. McArdle WD, Katch FI, Katch VI. Fisiologia do Exercício Nutrição, Energia e Desempenho Humano. $8^{\circ}$ ed. Rio de Janeiro: Guanabara Koogan; 2016.

\section{Acknowledgements}

This study was supported by Fundação de Amparo à Pesquisa do Estado de São Paulo (FAPESP\#2010/50048-1) and, in part, by Fundação Zerbini. MS. Graziela Amaro-Vicente, Dr. Mateus C. Laterza and Dr. Daniel G. Martinez were supported by Coordenação de Aperfeiçoamento de Pessoal de Nível Superior-CAPES. Dra. Maria U.P.B. Rondon (CNPq \# 308851/2017-0) was supported by Conselho Nacional de Desenvolvimento Científico e Tecnológico (CNPq).

\section{Corresponding author}

Maria Urbana Pinto Brandão Rondon, $\mathrm{PhD}$

Escola de Educação Física e Esporte da Universidade de São Paulo, Av. Prof. Mello de Moraes, 65, São Paulo, SP, Brazil

Email: urbana@usp.br

Manuscript received on May 30, 2018

Manuscript accepted on November 16, 2018

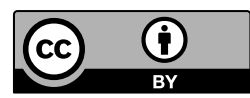

Motriz. The Journal of Physical Education. UNESP. Rio Claro, SP, Brazil - eISSN: 1980-6574 - under a license Creative Commons - Version 4.0 